\title{
Evaluation of the QuantaMatrix Multiplexed Assay Platform for Molecular Diagnosis of Multidrug- and Extensively Drug-Resistant Tuberculosis Using Clinical Strains Isolated in Myanmar
}

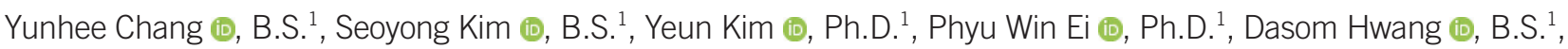 \\ Jongseok Lee $\mathbb{0}$, Ph.D. ${ }^{2}$, Chulhun L Chang $\left(\mathbb{0}\right.$, M.D., Ph.D. ${ }^{3}$, and Hyeyoung Lee $\mathbb{E}$, Ph.D. ${ }^{1}$ \\ ${ }^{1}$ Department of Biomedical Laboratory Science, College of Health Sciences, Yonsei University, Wonju, Korea; ${ }^{2}$ International Tuberculosis Research Center, \\ Changwon, Korea; ${ }^{3}$ Department of Laboratory Medicine, Pusan National University Yangsan Hospital, Yangsan, Korea
}

Background: Although the incidence of tuberculosis (TB) is decreasing, cases of multidrug-resistant (MDR) TB and extensively drug-resistant (XDR) TB continue to increase. As conventional phenotype drug susceptibility testing (pDST) takes six to eight weeks, molecular assays are widely used to determine drug resistance. we developed QuantaMatrix Multiplexed Assay Platform (QMAP) MDR/XDR assay (QuantaMatrix Inc., Seoul, Korea) that can simultaneously detect mutations related to both first- and second-line drug resistance (rifampin, isoniazid, ethambutol, fluoroquinolones, second-line injectable drugs, and streptomycin).

Methods: We used 190 clinical Mycobacterium tuberculosis (MTB) strains isolated from Myanmar, compared QMAP and pDST results, and determined concordance rates. Additionally, we performed sequence analyses for discordant results.

Results: QMAP results were 87.9\% (167/190) concordant with pDST results. In the 23 isolates with discordant results, the QMAP and DNA sequencing results completely matched.

Conclusions: The QMAP MDR/XDR assay can detect all known DNA mutations associated with drug resistance for both MDR- and XDR-MTB strains. It can be used for molecular diagnosis of MDR- and XDR-TB to rapidly initiate appropriate anti-TB drug therapy.

Key Words: Mycobacterium tuberculosis, Multidrug-resistant tuberculosis, Extensively drug-resistant tuberculosis, QuantaMatrix Multiplexed Assay Platform
Received: May 28, 2019

Revision received: August 6, 2019

Accepted: October 11, 2019

\section{Corresponding author:}

Hyeyoung Lee, Ph.D.

Department of Biomedical Laboratory

Science, College of Health Sciences, Yonsei

University, 1 Yeonsedae-gil, Wonju 26493,

Korea

Tel: +82-33-760-2740

Fax: +82-33-760-2561

E-mail: Hyelee@yonsei.ac.kr

\section{INTRODUCTION}

Tuberculosis (TB), caused by Mycobacterium tuberculosis (MTB) infection, is the ninth highest cause of death worldwide. There are an estimated 10 million cases of TB globally, with 1.6 million TB-associated deaths per year [1, 2]. Although the incidence of TB is decreasing by $2 \%$ every year, its prevalence and mortality rate remain high, necessitating comprehensive efforts for eradication [2].

Multidrug-resistant (MDR) TB and extensively drug-resistant (XDR) TB are of particular concern as they are difficult to treat. MDR-MTB exhibits resistance to two of the most important firstline drugs, rifampin (RIF) and isoniazid (INH), while XDR-MTB demonstrates resistance to RIF and $I \mathrm{NH}$, as well as to at least one fluoroquinolone $(\mathrm{FQ})$ and at least one second-line injectable drug (SLID; kanamycin, amikacin, and capreomycin) [3]. Treat- 
ment success rates for MDR-TB and XDR-TB are low, at 54\% and $30 \%$, respectively — and they are the main obstacles in TB eradication [1]. Therefore, rapid determination of the drug susceptibility of the TB-causing bacteria is important to ensure appropriate treatment.

Drug susceptibility testing (DST), used to select appropriate drugs, is a culture-based method and requires approximately six to eight weeks for completion [4]. To overcome the limitations of conventional assays, DST based on molecular diagnostic assays has been developed. For example, GenoType MTBDRplus (Hain Lifescience, Nehren, Germany) and GenoType MTBDRs/ (Hain Lifescience) are employed for the rapid identification of gene mutations related to MDR- and XDR-MTB using a line probe assay, while GeneXpert MTB/RIF (Cepheid AB, Solna, Sweden) is used to determine RIF resistance by real-time PCR [5].

Recently, QuantaMatrix Inc. (Seoul, Korea) developed the QuantaMatrix Multiplexed Assay Platform (QMAP), which utilizes magnetic micro-particles and a reverse hybridization assay. In QMAP, a probe for a specific gene is combined with a barcoded magnetic micro-particle, which is a carboxyl-functionalized magnetic disk with a 50- $\mu$ m-thick silica-coated surface and a graphical barcode that allows $>100$-plex coding capacity in high-throughput analysis [6]. Each probe enables the capture of PCR products with a complementary sequence and then emits fluorescence. A 100-plex capacity in a single microwell system allows the testing of 100 types of pathogens in one microwell with one sample. Previously, we developed an assay for detecting MDR-MTB based on QMAP and evaluated the utility of the assay using strains isolated from TB patients in Korea [7].

We have now developed a more sophisticated QMAP MDR/ XDR assay by adding a probe to identify genetic mutations associated with resistance to ethambutol (EMB), streptomycin (SM), FQ, and SLID in addition to RIF and INH. This molecular assay can simultaneously detect MDR- and XDR-MTB within six hrs. We evaluated the utility of this assay using clinical strains isolated from TB patients in Myanmar, which is among the 22 countries with the highest TB burden and is included in the global list of 27 countries with a high incidence of MDR-TB [8].

\section{METHODS}

\section{Clinical isolates}

A total of 190 MTB strains isolated from sputum samples of patients were collected from the National Tuberculosis Reference Laboratory (NTRL) in Yangon and the Upper Myanmar TB Lab- oratory (UMTL) in Patheingyi, Myanmar, from 2015 to 2016. Samples from patients suspected of having MDR-TB (113 from NTRL, and 77 from UMTL) were tested with the GeneXpert MTB/RIF assay, and positive samples were cultured in Ogawa egg slant medium to isolate MTB strains. This retrospective study was approved by the Ethics Review Committee of the Department of Medical Research in Yangon, Myanmar (Ethics/ DMR/2016/101).

\section{DNA extraction from clinical isolates}

Genomic DNA was extracted at the International Tuberculosis Research Center (ITRC, Changwon, Korea) using a simple boiling method with some modifications [9]. Briefly, the cultured colonies were suspended in $1 \mathrm{~mL}$ distilled water in an Eppendorf tube using a loop and heated at $99^{\circ} \mathrm{C}$ for 20 minutes with vortexing at 5 minutes intervals. The tube was then centrifuged at 12,000 $\times g$, $23^{\circ} \mathrm{C}$ for 5 minutes and the supernatant was removed and stored at $4^{\circ} \mathrm{C}$ until used in the QMAP MDR/XDR assay.

\section{OMAP MDR/XDR assay}

Three oligonucleotide probes specific to the genus Mycobacterium and 65 drug resistance-related gene probes were synthesized to detect MDR- and XDR-MTB (Table 1). Each probe was combined with a carboxyl-functionalized magnetic microdisk (QuantaMatrix Inc.). To amplify 11 target areas simultaneously, primers specific to biotin-attached species-specific areas and drug resistance-related areas were prepared and used for multiplex PCR. The PCR reactions consisted of $10 \mu \mathrm{L}$ of AccuPower Multiplex PCR PreMix (Bioneer, Daejeon, Korea), $5 \mu \mathrm{L}$ of the primer mixture, $1 \mu \mathrm{L}$ of internal control, and $2 \mu \mathrm{L}$ of molecular biology-grade water (GE Healthcare Life Sciences Korea, Seoul, Korea). PCR conditions were as follows: the mixture was denatured at $94^{\circ} \mathrm{C}$ for 5 minutes, followed by 45 cycles of $94^{\circ} \mathrm{C}$ for 20 seconds, $65^{\circ} \mathrm{C}$ for 1 minute, and $72^{\circ} \mathrm{C}$ for 5 minutes. The products were denatured at $25^{\circ} \mathrm{C}$ for 5 minutes by adding 10 $\mu \mathrm{L}$ of $2 \times$ denaturation solution (QuantaMatrix Inc.) to $10 \mu \mathrm{L}$ of the biotinylated PCR products. The resulting solution was diluted with $50 \mu \mathrm{L}$ of hybridization solution and dispensed onto a glass MatriPlate (Brooks, Chelmsford, MA, USA). The denatured (single-stranded) PCR products were combined with the probe attached to the microdisk with INCUBATOR-micro mixer (FINEPCR, Gunpo, Korea) at $650 \mathrm{rpm}$ and $35^{\circ} \mathrm{C}$ for 30 minutes. The microdisks were washed three times with $100 \mu \mathrm{L}$ of washing buffer (QuantaMatrix Inc.) with shaking at $650 \mathrm{rpm}, 25^{\circ} \mathrm{C}$ for 1 minute and then treated with staining buffer (QuantaMatrix Inc.) at $25^{\circ} \mathrm{C}$ and $650 \mathrm{rpm}$ for 10 minutes. The microdisks were 
Table 1. Target genes and regions of the probes used to detect mycobacteria and their resistance to specific drugs

\begin{tabular}{lcclc}
\hline Purposes & Drugs & $\begin{array}{l}\text { Target } \\
\text { genes }\end{array}$ & \multicolumn{1}{c}{ Target regions } & Probes (N) \\
\hline ID & - & rpoB & Codons 302-420 & 3 \\
DST & RIF & rpoB & Codons 504-533 & $11^{*}$ \\
& INH & katG & Codons 315 & $4^{*}$ \\
& & inhA & 8-17 bp upstream promoter region & $6^{*}$ \\
& EMB & embB & Codons 306 & 3 \\
& FQ & gyrA & Codons 88-94 & 10 \\
& gyrB & Codons 538-540 & 5 \\
& SLID & eis & 8-14, 37 bp upstream promotor region & 6 \\
& rrs & Bases 1400-1402, 1445, 1484 & 7 \\
& rpsL & Codons 4, 88 & 4 \\
& SM & rpsL & Bases 514-517 & 3 \\
\hline
\end{tabular}

*Adapted from Wang, et al. [7]. Copyright by Korean Society for Laboratory Medicine.

Abbreviations: ID, identification; RIF, rifampin; INH, isoniazid; EMB, ethambutol; FQ, fluoroquinolones; SLID, second-line injectable drugs; SM, streptomycin; DST, drug susceptibility testing.

washed three times with $100 \mu \mathrm{L}$ of washing buffer (QuantaMatrix Inc.) at $25^{\circ} \mathrm{C}$ for 1 minute, and the fluorescence intensity of each microdisk was automatically measured using the supplied software (QuantaMatrix Inc.).

\section{Phenotypic DST}

Phenotypic DST (pDST) for 12 first-line and second-line drugs was performed at ITRC using M-KIT plates (Korean Institute of Tuberculosis, Osong, Korea) with Löwenstein-Jensen medium, according to the manufacturer's protocol. The strains were transported to ITRC under the conditions of the materials transfer agreement. The assay was conducted using the modified absolute concentration method. The critical concentrations for each drug were as follows: INH, 0.2; RIF, 40; SM, 10.0; EMB, 2.0; kanamycin, 30; capreomycin, 40; cycloserine, 30; paraaminosalicylic acid, 1.0; ofloxacin, 4.0; moxifloxacin, 1.0; amikacin, 30; and levofloxacin, $2.0 \mu \mathrm{g} / \mathrm{mL}$.

\section{Concordance rates and DNA sequence analysis}

The results of QMAP and pDST were deemed concordant when the drug's QMAP result indicated the same resistance as that in its pDST. The target DNA sequences of samples with discordant results were analyzed using ABI Prism 3730xI DNA Sequencer (ThermoFisher Scientific Korea, Seoul, Korea) in Genotech (Daejeon, Korea) and compared with sequences in the NCBI GenBank database. The corresponding samples were se- quenced with designed primers for rpoB, katG, inh $A$, embB, gyrA, gyrB, eis, rrs, and rpsL.

\section{RESULTS}

pDST results of the clinical isolates and concordance between QMAP and pDST results

Table 2 shows the pDST results of all clinical isolates. Of the 190 isolates, 71 were MDR strains (37.4\%), 56 were susceptible to all tested drugs (29.5\%), 32 were pre-XDR strains (16.8\%), 15 were XDR strains (8.4\%), and seven were resistant only to INH (3.7\%).

The results for the 56 pan-susceptible isolates and seven isolates resistant only to INH showed a concordance rate of $96.4 \%$ and 100\%, respectively (Table 2). The results for MDR-MTB, pre-XDR-MTB, and XDR-MTB isolates showed concordance rates of $91.6 \%, 78.1 \%$, and $68.8 \%$, respectively. The results for the other nine isolates showed a concordance rate of $55.6 \%$. QMAP was able to detect 101 of 118 MDR- or XDR-MTB and pre-XDR-MTB isolates (85.6\%) or 113 of 134 any-drug-resistant MTB isolates (84.3\%).

\section{Concordance rates for the susceptibility and resistance patterns of each drug}

The QMAP and pDST concordance rates in each drug are shown in Table 3. Of the 70 RIF-susceptible and 120 RIF-resistant isolates, $95.7 \%$ and $96.7 \%$ showed concordant results using QMAP assay, respectively. The results for all $61 \mathrm{INH}$-susceptible isolates and $94.6 \%$ of INH-resistant isolates were concordant. Similarly, 96.7-100\% of the EMB-, FQ-, SLID-, and SM-susceptible isolates were detected using QMAP. However, the detection rates of the resistant isolates were slightly lower with QMAP; 56.5\% EMB-, 88.6\% FQ-, 75.0\% SLID-, and 90.6\% SM-resistant strains were detected.

\section{DNA sequencing of isolates with discordant results}

The 23 isolates showing discordant QMAP and qDST results were subjected to sequence analysis of the respective gene target region using the same primers (Table 4). Of the six MDR isolates identified by pDST, two showed no resistance; two, RIF resistance only; and two, INH resistance only in QMAP. Of the seven pre-XDR strains identified by $\mathrm{pDST}$, six had neither $\mathrm{FQ}$ nor SLID resistance and one was susceptible to INH in QMAP. Four XDR isolates identified by pDST showed pre-XDR genotypes in QMAP. For all these results, the sequence analysis results completely matched QMAP results (Table 4). 
Table 2. Comparison of drug susceptibility patterns between QMAP MDR/XDR assay and phenotypic DST results

\begin{tabular}{lccc}
\hline Result & $\begin{array}{c}\text { QMAP MDR/XDR } \\
(\mathrm{N})^{*}\end{array}$ & $\begin{array}{c}\text { Phenotypic DST } \\
(\mathrm{N})\end{array}$ & $\begin{array}{c}\text { Concordance } \\
\text { rate (\%) }\end{array}$ \\
\hline Pan-susceptible & 54 & 56 & 96.4 \\
Resistant to INH only & 7 & 7 & 100.0 \\
MDR & 65 & 71 & 91.6 \\
Pre-XDR & 25 & 32 & 78.1 \\
XDR & 11 & 15 & 73.3 \\
Others $\dagger$ & 5 & 9 & 55.6 \\
Total & 167 & 190 & 87.9 \\
\hline
\end{tabular}

*Indicates the number of isolates showing the expected results in accordance with the phenotypic DST results; ${ }^{\dagger}$ Three isolates were resistant to only $\mathrm{SM}$; three were resistant to INH and SM; one was resistant to RIF and SM; one was resistant to RIF, EMB, and SM; and one was resistant to INH, EMB, fluoroquinolones, second-line injectable drugs, and SM.

Abbreviations: DST, drug susceptibility testing; QMAP, QuantaMatrix Multiplexed Assay Platform; SM, streptomycin; RIF, rifampin; INH, isoniazid; $\mathrm{EMB}$, ethambutol; MDR, multidrug resistant; XDR, extensively drug resistant.

Table 3. Comparison of QMAP MDR/XDR assay and phenotypic DST results for each drug

\begin{tabular}{|c|c|c|c|c|c|c|}
\hline \multirow{2}{*}{ Drug } & \multicolumn{2}{|c|}{$\begin{array}{l}\text { QMAP assay } \\
\text { (Isolates, N) }\end{array}$} & \multicolumn{2}{|c|}{$\begin{array}{c}\text { Phenotypic DST } \\
\text { (Isolates, N) }\end{array}$} & \multicolumn{2}{|c|}{$\begin{array}{c}\text { Concordance rate } \\
(\%)\end{array}$} \\
\hline & $S$ & $\mathrm{R}$ & $S$ & $R$ & $S$ & $R$ \\
\hline RIF & 67 & 116 & 70 & 120 & 95.7 & 96.7 \\
\hline INH & 61 & 122 & 61 & 129 & 100.0 & 94.6 \\
\hline EMB & 82 & 67 & 82 & 108 & 100.0 & 56.5 \\
\hline $\mathrm{FQ}$ & 141 & 39 & 146 & 44 & 96.7 & 88.6 \\
\hline SLID & 170 & 15 & 170 & 20 & 100.0 & 75.0 \\
\hline SM & 62 & 116 & 62 & 128 & 100.0 & 90.6 \\
\hline
\end{tabular}

Abbreviations: S, susceptible; R, resistant; DST, drug susceptibility testing; QMAP, QuantaMatrix Multiplexed Assay Platform; MDR, multidrug resistant; $X D R$, extensively drug resistant; RIF, rifampin; INH, isoniazid; EMB, ethambutol; FQ, fluoroquinolones; SLID, second-line injectable drugs; SM, streptomycin.

\section{DISCUSSION}

The utility of the QMAP assay was evaluated using MTB strains isolated from TB patients. We also compared the results of QMAP and pDST; the assays showed an overall concordance rate of $87.9 \%$. The DNA sequencing results of the 23 isolates with discordant results matched QMAP results. Because of the inherent limitation of molecular DST methods, not all strains resistant to a specific drug can be detected as many drug resistance genes remain to be identified. The purpose of molecular DST is timely detection of resistant strains as many as possible.
Table 4. Sequence analysis of 23 isolates with discordant QMAP assay and phenotypic DST results

\begin{tabular}{llll}
\hline QMAP MDR/XDR & \multicolumn{1}{c}{ Phenotypic DST } & $\begin{array}{c}\text { Gene sequences for } \\
\text { the discordant } \\
\text { results* }\end{array}$ & $\begin{array}{c}\text { Isolates } \\
\text { (N) }\end{array}$ \\
\hline SM-R & Pan-S & $\begin{array}{c}\text { rpsL 43 AAG } \rightarrow \text { AGG } \\
\text { rpsL 88 AAG } \rightarrow \text { AGG }\end{array}$ & 2 \\
Pan-S & MDR & rpoB, katG, inhA WT & 2 \\
RIF-R & MDR & katG, inhA WT & 2 \\
INH-R & MDR & ropB WT & 2 \\
MDR & Pre-XDR (FQ-R) & gyrA, gyrB WT & 4 \\
MDR & Pre-XDR (SLID-R) & eis, rrs, rpsL WT & 2 \\
RIF-, EMB-, FQ-R & Pre-XDR (FQ-R) & katG, inhA WT & 1 \\
Pre-XDR (SLID-R) & XDR & gyrA, gyrB WT & 1 \\
Pre-XDR (FQ-R) & XDR & eis, rrs, rpsL WT & 3 \\
Pan-S & SM-R & rpsL WT & 1 \\
RIF-R, SM-R & RIF-, EMB-, SM-R & embB WT & 1 \\
Pre-XDR (FQ-R) & INH-, EMB-, SM-R & rpoB 526 CAC $\rightarrow$ AAC & 1 \\
& & gyrA 94 GAC $\rightarrow$ TAC & \\
XDR & INH-, EMB-, FQ-, SLID-, & rpoB 533 CTG $\rightarrow$ CAG & 1 \\
& SM-R & embB WT & \\
\hline
\end{tabular}

*The target regions of each gene are described in Table 1.

Abbreviations: DST, drug susceptibility testing; QMAP, QuantaMatrix Multiplexed Assay Platform; RIF, rifampin; INH, isoniazid; FQ, fluoroquinolones; SM, streptomycin; SLID, second-line injectable drugs; Pan-S, pan-susceptible; MDR, multidrug resistant; XDR, extensively drug resistant; pre-XDR, MDR with FQ resistance or MDR with resistance to SLID; WT, wild type; $\mathrm{EMB}$, ethambutol.

In that context, our current assay could detect $87.9 \%$ of MDRand XDR-MTB isolates accurately and rapidly.

Currently, several molecular DST assays, such as the AdvanSure MDR-TB GenoBlot assay kit (LG Chem, Seoul, Korea) and GenoType MTBDRplus, are clinically used. Both kits demonstrated good performance for MDR-MTB detection in clinical isolates, with concordance rates of $94.3 \%$ and $88.5-98.2 \%$ [10-12], respectively. One study reported that the results of GenoType MTBDRsl, a kit for XDR-MTB detection, showed a concordance rate of $94.7 \%$ [13]. However, a pooled analysis reported lower sensitivity of 75-80\%, accompanied by high specificity of $91-100 \%$, for detection of XDR-MTB isolates [14]. Our discordant PDST and QMAP results might have occurred for the following reasons. First, not all mutations associated with drug resistance are known [15]. Currently, only approximately 95\% of RIF resistance due to the rpoB gene, which contains the RIF resistance determining region (RRDR), is detectable [16]; in case of kat $G$ and inhA, which are genes related to $I N H$ resistance, the detection rates of INH resistance have been reported 
to be $70 \%$ and $10 \%$, respectively [17]; and for the gyrA and gyr $B$ regions associated with $F Q$ resistance, the detection rates is only approximately $60 \%$ [18]. Second, the tested isolates possibly exhibited heteroresistance. Heteroresistance is detected in $20-30 \%$ of TB patients and can be caused by a mixed infection of two different isolates or by acquisition of drug resistance during treatment [19-21]. Patients infected with heteroresistant isolates might convert to full resistance [22]. In such cases, $\leq 1 \%$ of the DNA of resistant isolates could be detected by molecular methods [23]. Third, isolates with low-level resistance and borderline resistance exhibiting susceptibility in pDST could be detected by molecular methods [24]. For these reasons, although molecular assays might not replace the pDST, they could be more widely used for detecting drug resistance, as WHO has recently reported [25].

The following study limitations should be considered. First, the QMAP MDR/XDR assay results showed low concordance (56.5\%) with pDST results for detecting EMB resistance. This low detection rate could be attributed to a lack of appropriate probes. EMB resistance occurs most frequently at codons 306 , 406 , and 497 in the embB gene [26, 27]; however, we designed probes related only to codon 306 . Second, this study was conducted using only selected strains, including a high proportion of drug-resistant strains, and the performance evaluation was mainly focused on the detection of resistance. However, in the real situation, there would be a larger proportion of susceptible strains, potentially leading to false-positive detection of resistance. Therefore, further studies using different and a greater number of probes and including an adequate number of susceptible strains are needed to improve the performance of QMAP MDR/XDR assay and confirm its specificity.

As not all types of mutations that cause drug resistance have been identified, using molecular assays in conjunction with pDST could enable the rapid and accurate determination of drug susceptibility of MTB isolates, thereby facilitating timely initiation of appropriate anti-TB drug therapy [28]. In particular, the $100 \%$ concordance between DNA sequencing and QMAP results indicates that QMAP could detect all known, or at least, targeted DNA mutations associated with drug resistance. Therefore, the QMAP MDR/XDR assay can be used for molecular diagnosis of MDR- and XDR-TB.

\section{Acknowledgements}

The Korea International Cooperation Agency (KOICA) has supported TB eradication in Myanmar through activities such as the establishment of the Advanced Molecular Research Center within the Department of Medical Research of Myanmar. The isolates were collected during such collaborative studies and were kindly provided to our research team.

\section{Author Contributions}

All authors participated in developing and evaluating the QMAP MDR/XDR assay in collaboration with QuantaMatrix Inc.

\section{Conflicts of Interest}

The QMAP MDR/XDR assay was developed and evaluated in collaboration with QuantaMatrix Inc. No other potential conflicts of interest relevant to this article were reported by the authors.

\section{Research Funding}

This study was supported by the National Research Foundation of Korea (NRF-2016S1A5B8925203).

\section{ORCID}

Yunhee Chang https://orcid.org/0000-0003-1857-1955 Seoyong Kim https://orcid.org/0000-0002-0071-3954

Yeun Kim

Phyu Win Ei

Dasom Hwang

Jongseok Lee

Chulhun L Chang

https://orcid.org/0000-0002-5584-3318

https://orcid.org/0000-0002-2486-3260

https://orcid.org/0000-0002-3426-8932

https://orcid.org/0000-0002-4340-9856

https://orcid.org/0000-0001-9117-4919

Hyeyoung Lee

https://orcid.org/0000-0003-1572-5250

\section{REFERENCES}

1. WHO. Global tuberculosis report 2017. https://www.who.int/tb/publications/global_report/gtbr2017_main_text.pdf (Updated on Aug 2019).

2. WHO. Global tuberculosis report 2018. http://apps.who.int/iris/bitstream/handle/10665/274453/9789241565646-eng.pdf (Updated on Aug 2019).

3. WHO. Definitions and reporting framework for tuberculosis-2013 revision. https://apps.who.int/iris/bitstream/handle/10665/79199/9789241505345_ eng.pdf (Updated on Aug 2019).

4. Choi J, Yoo J, Kim KJ, Kim EG, Park KO, Kim H, et al. Rapid drug susceptibility test of Mycobacterium tuberculosis using microscopic timelapse imaging in an agarose matrix. Appl Microbiol Biotechnol 2016;100: 2355-65.

5. Pang $\mathrm{Y}$, Dong $\mathrm{H}$, Tan $\mathrm{Y}$, Deng $\mathrm{Y}$, Cai $\mathrm{X}$, Jing $\mathrm{H}$, et al. Rapid diagnosis of MDR and XDR tuberculosis with the MeltPro TB assay in China. Sci Rep 2016;6:25330. 
6. Kim LN, Kim M, Jung K, Bae HJ, Jang J, Jung Y, et al. Shape-encoded silica microparticles for multiplexed bioassays. Chem Commun (Camb) 2015;51:12130-3.

7. Wang HY, Uh Y, Kim S, Cho E, Lee JS, Lee H. Detection of rifampicinand isoniazid-resistant Mycobacterium tuberculosis using the QuantaMatrix Multiplexed Assay Platform system. Ann Lab Med 2018;38:56977.

8. Han ET, Lee JS, Cheong JH, Chang CL, Nyunt MH, Aung WW, et al. Current status of standard diagnostics and treatment for malaria, tuberculosis, and hepatitis in Myanmar. Lab Med Online 2017;7:94-102.

9. Jensen MA, Webster JA, Straus N. Rapid identification of bacteria on the basis of polymerase chain reaction-amplified ribosomal DNA spacer polymorphisms. Appl Environ Microbiol 1993;59:945-52.

10. Kim J, Park YJ, Lee NY, Chang CL, Lee M, Shin JH. Evaluation of the AdvanSure MDR-TB GenoBlot assay for detection of rifampin and isoniazid resistant Mycobacterium tuberculosis complex in respiratory specimens. Korean J Clin Microbiol 2012;15:117-24.

11. Maurya AK, Umrao J, Singh AK, Kant S, Kushwaha RA, Dhole TN. Evaluation of GenoType MTBDRplus assay for rapid detection of drug susceptibility testing of multi-drug resistance tuberculosis in Northern India. Indian J Pathol Microbiol 2013;56:139-43.

12. Bedewi Omer Z, Mekonnen Y, Worku A, Zewde A, Medhin G, Mohammed T, et al. Evaluation of the GenoType MTBDRplus assay for detection of rifampicin- and isoniazid-resistant Mycobacterium tuberculosis isolates in central Ethiopia. Int J Mycobacteriol 2016;5:475-81.

13. Tagliani E, Cabibbe AM, Miotto P, Borroni E, Toro JC, Mansjo M, et al. Diagnostic performance of the new version (v2.0) of GenoType MTBDRs/ assay for detection of resistance to fluoroquinolones and second-line injectable drugs: a multicenter study. J Clin Microbiol 2015;53:2961-9.

14. WHO. The use of molecular line probe assays for the detection of resistance to second-line anti-tuberculosis drugs: policy guidance. WHO HTM/TB/2016.07. http://www.who.int/iris/bitstream/10665/246131/1/ 9789241510561-eng.pdf (Updated on Aug 2019).

15. Laurenzo D and Mousa SA. Mechanisms of drug resistance in Mycobacterium tuberculosis and current status of rapid molecular diagnostic testing. Acta Trop 2011;119:5-10.

16. Lee AS, Lim IH, Tang LL, Wong SY. High frequency of mutations in the rpoB gene in rifampin-resistant clinical isolates of Mycobacterium tuberculosis from Singapore. J Clin Microbiol 2005;43:2026-7.

17. Dookie N, Rambaran S, Padayatchi N, Mahomed S, Naidoo K. Evolution of drug resistance in Mycobacterium tuberculosis: a review on the molecular determinants of resistance and implications for personalized care. J Antimicrob Chemother 2018;73:1138-51.

18. Chien JY, Chiu WY, Chien ST, Chiang CJ, Yu CJ, Hsueh PR. Mutations in gyrA and gyrB among fluoroquinolone- and multidrug-resistant $M y$ - cobacterium tuberculosis isolates. Antimicrob Agents Chemother 2016; 60:2090-6.

19. Hofmann-Thiel S, van Ingen J, Feldmann K, Turaev L, Uzakova GT, Murmusaeva G, et al. Mechanisms of heteroresistance to isoniazid and rifampin of Mycobacterium tuberculosis in Tashkent, Uzbekistan. Eur Respir J 2009;33:368-74.

20. Chakravorty S, Aladegbami B, Thoms K, Lee JS, Lee EG, Rajan V, et al. Rapid detection of fluoroquinolone-resistant and heteroresistant $\mathrm{Myco}$ bacterium tuberculosis by use of sloppy molecular beacons and dual melting-temperature codes in a real-time PCR assay. J Clin Microbiol 2011;49:932-40.

21. Shin SS, Modongo C, Baik Y, Allender C, Lemmer D, Colman RE, et al. Mixed Mycobacterium tuberculosis-strain infections are associated with poor treatment outcomes among patients with newly diagnosed tuberculosis, independent of pretreatment heteroresistance. J Infect Dis 2018;218:1974-82.

22. Mekonnen D, Admassu A, Mulu W, Amor A, Benito A, Gelaye W, et al. Multidrug-resistant and heteroresistant Mycobacterium tuberculosis and associated gene mutations in Ethiopia. Int J Infect Dis 2015;39:348.

23. Liang B, Tan Y, Li Z, Tian X, Du C, Li H, et al. Highly sensitive detection of isoniazid heteroresistance in Mycobacterium tuberculosis by DeepMelt assay. J Clin Microbiol 2018;56:e01239-17.

24. Ocheretina O, Escuyer VE, Mabou MM, Royal-Mardi G, Collins S, Vilbrun SC, et al. Correlation between genotypic and phenotypic testing for resistance to rifampin in Mycobacterium tuberculosis clinical isolates in Haiti: investigation of cases with discrepant susceptibility results. PLoS One 2014;9:e90569.

25. WHO. The use of next-generation sequencing technologies for the detection of mutations associated with drug resistance in Mycobacterium tuberculosis complex: technical guide. WHO CDS/TB/2018.19. https:// apps.who.int/iris/bitstream/handle/10665/274443/WHO-CDS-TB2018.19-eng.pdf (Updated on Aug 2019).

26. Khosravi AD, Sirous M, Abdi M, Ahmadkhosravi N. Characterization of the most common embCAB gene mutations associated with ethambutol resistance in Mycobacterium tuberculosis isolates from Iran. Infect Drug Resist 2019;12:579-84.

27. Sun $Q$, Xiao TY, Liu HC, Zhao XQ, Liu ZG, Li YN, et al. Mutations within embCAB are associated with variable level of ethambutol resistance in Mycobacterium tuberculosis isolates from China. Antimicrob Agents Chemother 2017;62:e01279-17.

28. Gkaravela L, Papadimitriou-Olivgeris M, Foka A, Kolonitsiou F, Spiliopoulou $\mathrm{A}$, Charokopos $\mathrm{N}$, et al. Combination of commercially available molecular assays and culture based methods in diagnosis of tuberculosis and drug resistant tuberculosis. Braz J Microbiol 2017;48:785-90. 